Société d'histoire de la révolution de 1848 et des

révolutions du XIXe siècle

$45 \mid 2012$

Le quotidien des techniques

\title{
Hélène BLAIS, Florence DEPREST et Pierre SINGARAVÉLOU, Territoires impériaux. Une histoire spatiale du fait colonial
}

Paris, Publications de la Sorbonne, 2011, 336 p. ISBN : 978-2-85944-675-8.

28 euros.

Luis Teixeira

\section{(2) OpenEdition}

Journals

Édition électronique

URL : http://journals.openedition.org/rh19/4412

DOI : 10.4000/rh19.4412

ISSN : $1777-5329$

Éditeur

La Société de 1848

Édition imprimée

Date de publication : 31 décembre 2012

Pagination : 226-228

ISSN : 1265-1354

Référence électronique

Luis Teixeira, « Hélène BLAIS, Florence DEPREST et Pierre SINGARAVÉLOU, Territoires impériaux. Une histoire spatiale du fait colonial », Revue d'histoire du XIXe siècle [En ligne], 45 | 2012, mis en ligne le 02 avril 2013, consulté le 22 septembre 2020. URL : http://journals.openedition.org/rh19/4412 ; DOI : https://doi.org/10.4000/rh19.4412

Ce document a été généré automatiquement le 22 septembre 2020.

Tous droits réservés 


\section{Hélène BLAIS, Florence DEPREST et Pierre SINGARAVÉLOU, Territoires impériaux. Une histoire spatiale du fait colonial}

Paris, Publications de la Sorbonne, 2011, 336 p. ISBN : 978-2-85944-675-8. 28 euros.

Luis Teixeira

\section{RÉFÉRENCE}

Hélène BLAIS, Florence DEPREST et Pierre SINGARAVÉLOU, Territoires impériaux. Une histoire spatiale du fait colonial, Paris, Publications de la Sorbonne, 2011, 336 p. ISBN : 978-2-85944-675-8. 28 euros.

1 Géographie et colonisation : le thème s'est récemment imposé en France, et l'on peut se réjouir de la multiplication des publications, individuelles et collectives, qui participent au rajeunissement salutaire de l'histoire coloniale. Mais Territoires impériaux n'est pas seulement le dernier rejeton de cette vague montante. Il est de ces livres que leur sujet établit de facto en référence incontournable. De ces bornes qui délimitent un champ d'étude et indiquent de nouvelles pistes de recherche. Rien de moins. Ce recueil de onze études inédites renferme, en sus, une réflexion de synthèse fort bienvenue et une bibliographie courte (neuf pages), mais complète. La succession des titres déroule l'histoire d'un champ historiographique qui a su tirer avantage d'une interdisciplinarité engagée et pragmatique, portée par un dialogue toujours plus animé entre chercheurs du monde entier. Il faut savoir gré aux auteurs, et en particulier à Hélène Blais et à Florence Deprest, les coordinatrices du programme de recherche dont est issu ce recueil, d'ouvrir au public français cette fenêtre sur une histoire impériale en train de se faire, aujourd'hui, en France et dans le monde. 
2 Histoire coloniale, histoire impériale : un tour de passe-passe lexical ? Bien plus, et ce livre aide à le comprendre. L'empire, c'est d'abord une échelle d'analyse, qui parvient à commuer en dialectique l'opposition souvent préjugée entre l'échelle micro du terrain colonial et l'échelle macro du rapport complexe entre métropole et colonies. Une échelle, cet empire, donc. Mais pas seulement. Comme espace, l'empire se distingue de la colonie par une certaine plasticité. La colonie : territoire politique et administratif, territoire aux limites (en principe) définies et maîtrisées - en bref, territoire "pacifié " (comme disent les sources coloniales). L'empire : un work in progress, un projet sans cesse redéfini de territorialisation militaire, politique, économique et culturelle. Réinscrire l'histoire du «fait colonial » dans l'espace, c'est dépasser le récit lisse et familier de la conquête coloniale. En effet, le processus d'avènement d'un territoire impérial n'est pas réductible à la prise de possession officielle d'un territoire déjà fait. Sa création, sa structuration est une entreprise de longue haleine, qui mobilise notamment les géographes et les savoirs géographiques des puissances coloniales. C'est précisément la fonction de ces derniers qui est interrogée par les contributeurs de ce volume.

3 Territoires impériaux propose une expertise $\mathrm{du}$ "fait colonial» qui esquive les généralisations, en multipliant les études de cas très fouillées. En témoigne le plan de l'ouvrage. La première partie, intitulée "Savoirs, inventaires, catégories ", s'intéresse à la production du savoir et des savoir-faire géographiques sur le terrain colonial. La deuxième partie, "Cultures visuelles», explore les modalités de présentation géographique des empires. Deux média sont privilégiés : le voyage d'étude et la carte. Les contributeurs enquêtent sur l'efficace de chaque médium, sa fonction performative, et proposent, pour finir, l'heureuse notion de "culture visuelle des empires ». La troisième et dernière partie, "Espaces, territoires, lieux: des productions impériales? ", étudie avec minutie la production d'espaces spécifiquement coloniaux, à l'instar des concessions en Chine et des stations d'altitude en Indochine.

4 Il ressort de la lecture de cette belle série d'études la pertinence de l'analyse microhistorique pour l'histoire impériale. Ce type d'analyse permet d'écrire une " histoire au ras du sol colonial», qui dévoile les traces et ressuscite les gestes concrets de la production de savoir géographique en situation coloniale. L'historien y gagne ainsi une visibilité multiscalaire sur les processus élaborés sur le terrain et leur interprétation par les institutions impériales de la métropole. En effet, dans le désert saharien, dans la forêt dense du Congo, au Soudan entre Tchad et Niger, en Chine, sur les hauts plateaux indochinois, dans la cordillère des Andes, partout, le chercheur retrouve les agents de la colonisation européenne, cumulant les titres d'autorité et les identités sociales (savants et/ou militaires et/ou administrateurs, etc.), et s'affairant à négocier leur présence sur des territoires nouveaux pour eux. Car ce qui se joue, dans ces «coins perdus » d'Afrique, d'Asie et d'Amérique, n'est rien de moins que l'existence toujours recommencée des "territoires impériaux», dans une dialectique constante entre les échelles locale, coloniale et impériale. Inventions, circulations, distributions, applications de savoirs et de savoir-faire sur le territoire colonisé. Mais aussi: hybridations, ajustements, partages imposés ou argumentés par le colonisateur. Car les Européens doivent (bien sûr) compter avec les populations locales, qui elles aussi possèdent leurs propres représentations, organisations et instruments d'appropriation $\mathrm{du}$ territoire. Et les transferts de savoirs et de pouvoirs sont si nombreux, si subtils, si labiles aussi, que l'historien doit travailler les sources, généralement d'origine 
européenne, avec d'infinies précautions, qu'une érudition critique peut seule garantir. L'historien reconstitue réseaux d'acteurs et chaînes d'informations, exhume notes et croquis de terrain, jusqu'aux cartes majestueuses dressées et diffusées par les institutions impériales de la métropole.

5 On doit lire Territoires impériaux à l'aune de son introduction, signée par ses trois directeurs, et dont le titre sonne comme un manifeste : «Pour une histoire spatiale du fait colonial ». Après avoir bien défini l'état de la question, les auteurs justifient, sur la fin, l'économie de l'ouvrage. Entre les deux, un exposé de méthode étonnamment succinct - deux pages à peine (p.12-13) - auquel il manque, à notre avis, une réflexion plus approfondie sur le parti que l'histoire impériale pourra tirer des concepts et des méthodes d'une géographie contemporaine riche de réflexivité critique. Carence d'autant plus regrettable que le ressort même de cette recherche était précisément d'éclairer les emplois scientifiques et politiques de la géographie dans la fabrication territoriale des empires. Ces dernières remarques n'enlèvent cependant rien à la valeur de l'ouvrage, qui devrait compter pour les historiens des savoirs en situation coloniale et des colonisations en général. 